\title{
SOME THEOREMS ON STIRLING-TYPE PAIRS
}

\author{
by LEETSCH C. HSU
}

(Received 25th March 1992)

In memory of the 300th anniversary of James Stirling's birth

\begin{abstract}
It is shown that an extended Schlömilch formula for Stirling-type pairs of numbers and the inversion formula of Lagrange are implied by each other. Also proved are some congruence relations modulo a prime number $p(>2)$ associated with generalized Stirling numbers. The third result is concerned with the asymptotic expansions of Stirling-type pairs involving large parameters.
\end{abstract}

1991 Mathematics subject classification: 05A99, 10A40, 11B73, 41A60.

\section{Introduction}

By a Stirling-type pair (or generalized Stirling number pair) we mean a pair of two-parameter numbers $A_{1}(n, k)$ and $A_{2}(n, k)$ that are defined by the formal expansions

$$
\frac{(f(t))^{k}}{k !}=\sum_{n \geqq 0} A_{1}(n, k) \frac{t^{n}}{n !}, \quad \frac{(g(t))^{k}}{k !}=\sum_{n \geqq 0} A_{2}(n, k) \frac{t^{n}}{n !},
$$

where $f(t)=\sum_{k \geqq 1} a_{k} t^{k}$ and $g(t)=\sum_{k \geqq 1} b_{k} t^{k}$ are reciprocal formal power series over the complex field $\overline{\mathbf{C}}$ with $f(0)=g(0)=0$ and $a_{1}=b_{1}=1$, namely $f \circ g(t)=t$. Note that $A_{1}(n, k)=A_{2}(n, k)=0$ for $n<k$, and that $A_{1}(0,0)=A_{2}(0,0)=1$. $t=0$ :

Evidently, both $A_{1}(n, k)$ and $A_{2}(n, k)$ may be expressed by using formal derivatives at

$$
A_{1}(n, k)=\frac{1}{k !}\left(\frac{d}{d t}\right)_{0}^{n}(f(t))^{k}, \quad A_{2}(n, k)=\frac{1}{k !}\left(\frac{d}{d t}\right)_{0}^{n}(g(t))^{k}
$$

In case the reciprocal series (compositional inverse) $g$ of $f$ is not easily determined, one may compute $A_{2}(n, k)$ by using the expression

$$
A_{2}(n, k)=\left(\begin{array}{l}
n-1 \\
k-1
\end{array}\right)\left(\frac{d}{d t}\right)_{0}^{n-k}\left(\frac{t}{f(t)}\right)^{n}
$$


In fact, this expression is a direct consequence of Lagrange's inversion formula which will be recalled in the next section.

It is known that for every Stirling-type pair $\left\{A_{1}(n, k), A_{2}(n, k)\right\}$ there hold the inverse relations

$$
x_{n}=\sum_{k=0}^{n} A_{1}(n, k) y_{k} \Leftrightarrow y_{n}=\sum_{k=0}^{n} A_{2}(n, k) x_{k}
$$

with their rotated form

$$
x_{n}=\sum_{k \geqq n} A_{1}(k, n) y_{k} \Leftrightarrow y_{n}=\sum_{k \geqq n} A_{2}(k, n) x_{k}
$$

where, in (5) one has to assume that either $\left\{x_{k}\right\}$ or $\left\{y_{k}\right\}$ vanishes ultimately. For a more detailed exposition of Stirling-type pairs and their general properties (e.g. recurrence relations and convolution formulas, etc.), refer to our earlier paper [6] (cf. also [7]).

Examples. In principle one can construct Stirling-type pairs as many as one likes. The following is a table of various special pairs easily justified by observation or simple computations (cf. [6]).

\begin{tabular}{c|c|c|c}
$f(t)$ & $g(t)$ & $A_{1}(n, k)$ & $A_{2}(n, k)$ \\
\hline $\log (1+t)$ & $e^{t}-1$ & $S_{1}(n, k)$ & $S_{2}(n, k)$ \\
$\tan t$ & $\arctan t$ & $T_{1}(n, k)$ & $T_{2}(n, k)$ \\
$\sin t$ & $\arcsin t$ & $\sigma_{1}(n, k)$ & $\sigma_{2}(n, k)$ \\
$2 \operatorname{arcsinh} \frac{t}{2}$ & $2 \sinh \frac{t}{2}$ & $t_{1}(n, k)$ & $t_{2}(n, k)$ \\
$\tanh t$ & $\operatorname{arctanh}$ & $\tau_{1}(n, k)$ & $\tau_{2}(n, k)$ \\
$e^{\sin t}-1$ & $\arcsin (\log (1+t))$ & $\alpha_{1}(n, k)$ & $\alpha_{2}(n, k)$ \\
$e^{\arcsin t}-1$ & $\sin (\log (1+t))$ & $\beta_{1}(n, k)$ & $\beta_{2}(n, k)$ \\
$e^{\arctan t}-1$ & $\tan (\log (1+t))$ & $\gamma_{1}(n, k)$ & $\gamma_{2}(n, k)$ \\
$t /(t-1)$ & $t /(t-1)$ & $(-1)^{k} \frac{n !}{k !}\left(\begin{array}{l}n-1 \\
k-1\end{array}\right)$ & $(-1)^{k} \frac{n !}{k !}\left(\begin{array}{l}n-1 \\
k-1\end{array}\right)$
\end{tabular}

Here in the table $S_{1}(n, k)$ and $S_{2}(n, k)$ are known as the two kinds of ordinary Stirling numbers whose importance in the calculus of finite differences, combinatorial mathematics and numerical analysis is well-known. $T_{1}(n, k)$ and $T_{2}(n, k)$ are called tangent and arctangent numbers respectively, which have also applications in combinatorics (cf. [5]). Of particular interest are the central factorial numbers $t_{1}(n, k)$ and $t_{2}(n, k)$ whose importance and fruitfulness in applications have been explored recently by $P$. L. Butzer 
and his cooperators (cf. $[3,4]$ ). The last entry of the table is obviously related to Lah numbers.

For a given Stirling-type pair $\left\{A_{1}, A_{2}\right\}$ one may define two kinds of generalized Bell numbers as follows

$$
W_{i}(n):=\sum_{k=1}^{n} A_{i}(n, k),(n \geqq 1) ; \quad W_{i}(0):=1,(i=1,2) .
$$

Clearly the equations given by (1) lead immediately to the exponential generating functions

$$
e^{f(t)}=\sum_{n \geqq 0} W_{1}(n) \frac{t^{n}}{n !}, \quad e^{g(t)}=\sum_{n \geqq 0} W_{2}(n) \frac{t^{n}}{n !}
$$

Moreover, the recurrence relations for $W_{i}$ numbers $(i=1,2)$

$$
W_{i}(n+1)=\sum_{j=0}^{n}\left(\begin{array}{l}
n \\
j
\end{array}\right) A_{i}(n-j+1,1) W_{i}(j)
$$

can be deduced from the vertical recurrence relations for Stirling-type pairs (cf. [6])

$$
\left.A_{i}(n+1, k)=\sum_{j=0}^{n}\left(\begin{array}{l}
n \\
j
\end{array}\right) A_{i}(j, k-1) A_{i}(n-j)+1,1\right)
$$

where $A_{1}(j, 1)=f^{(j)}(0)$ and $A_{2}(j, 1)=g^{(j)}(0)$. Evidently the well-known exponential formula due to Bell

$$
e^{e^{t}-1}=\sum_{n \geqq 0} B_{n} \frac{t^{n}}{n !} \quad \text { with } \quad B_{n}=\sum_{k=1}^{n} S_{2}(n, k)
$$

is an instance of (7) with $g(t):=e^{t}-1, A_{2}:=S_{2}, W_{2}(n):=B_{n}$.

As is known, the Stirling number $S_{2}(n, k)$ can be expressed as a single summation of elementary terms, which are themselves products and quotients of factorials and powers. 
However, the shortest formula for $S_{1}(n, k)$ should consist of a double summation of elementary terms, so that in accordance with the terminology adopted in Comtet $[5, \S 5.7] S_{2}(n, k)$ and $S_{1}(n, k)$ are said to be of rank one and two, respectively. In fact, the following formula due to Schlömilch (1852) is known as the 'shortest formula' for $S_{1}(n, k)$ (cf. $\left.[5,10]\right)$ :

$$
\begin{aligned}
S_{1}(n, k) & =\sum_{v=0}^{n-k}(-1)^{v}\left(\begin{array}{c}
2 n-k \\
n-k-v
\end{array}\right)\left(\begin{array}{l}
n-1+v \\
n-k+v
\end{array}\right) S_{2}(n-k+v, v) \\
& =\sum_{v=0}^{n-k} \sum_{j=0}^{v} \frac{(-1)^{v-j}}{j !(v-j) !}\left(\begin{array}{c}
2 n-k \\
n-k-v
\end{array}\right)\left(\begin{array}{l}
n-1+v \\
n-k+v
\end{array}\right)(v-j)^{n-k+v}
\end{aligned}
$$

In the next section we will develop a general Schlömilch formula for Stirling-type pairs, and show its close connection with the inversion formula of Lagrange.

\section{The Schlömilch formula}

For any given formal power series $f(t)=\sum_{k \geqq 0} a_{k} t^{k}$ we denote

$$
\mathrm{C}_{t^{n}} f:=a_{n}=\text { the coefficient of } t^{n} \text { in } f(t) \text {. }
$$

The classical inversion formula of Lagrange may now be stated as follows: Let $f(t)$ be a formal series with $f(0)=0$ and $f^{\prime}(0) \neq 0$, and let $g(t)$ be its reciprocal series so that $g \circ f(t)=f \circ g(t)=t$. Then we have the formula (cf. $[5, \$ 3.8]$ )

$$
\mathbf{C}_{t^{n}}(g(t))^{k}=\frac{k}{n} \mathbf{C}_{t^{n-k}}\left(\frac{f(t)}{t}\right)^{-n}
$$

Also, let us recall a useful differentiation formula: For any given formal series $\phi(t)$ with $\phi(0)=1$ and any number $\alpha \in C$ we have (cf. also [5])

$$
\left(\frac{d}{d t}\right)_{0}^{n}(\phi(t))^{-\alpha}=\alpha\left(\begin{array}{c}
n+\alpha \\
n
\end{array}\right) \sum_{j=1}^{n} \frac{(-1)^{j}}{\alpha+j}\left(\begin{array}{c}
n \\
j
\end{array}\right)\left(\frac{d}{d t}\right)_{0}^{n}(\phi(t))^{j}
$$

Let $\left\{A_{1}(n, k), A_{2}(n, k)\right\}$ be a Stirling-type pair generated by (1). Then, starting with (2) and making use of (12) and (13) one can establish a general Schlömilch formula of the form

$$
A_{i}(n, k)=\sum_{v=0}^{n-k}(-1)^{v}\left(\begin{array}{c}
2 n-k \\
n-k-v
\end{array}\right)\left(\begin{array}{l}
n-1+v \\
n-k+v
\end{array}\right) A_{j}(n-k+v, v) .
$$


where the index pair $(i, j)$ may be either of $(1,2)$ and $(2,1)$, so that $(14)$ is a reciprocal relation between $A_{1}(\cdot, \cdot)$ and $A_{2}(\cdot, \cdot)$.

Theorem 1. Having assumed the differentiation formula (13), the general Schlömilch formula (14) and the Lagrange inversion formula (12) are equivalent, i.e. they are deducible from each other.

Proof. Recall that the pair of reciprocal formal series $f(t)$ and $g(t)$ associated with $\left\{A_{1}(n, k), A_{2}(n, k)\right\}$ have the property that $f(0)=g(0)=0$ and $f^{\prime}(0)=g^{\prime}(0)=1$. Consequently the formal series $\phi(t):=f(t) / t$ has the property $\phi(0)=1$. Hence for each $f$ the relation (12) may be rewritten as

$$
\mathbf{C}_{t^{n}}(g(t))^{k}=\frac{k}{n} \mathbf{C}_{t^{n-k}}(\phi(t))^{-n}
$$

Now applying the differentiation formula (13) to the right-hand side of (15), one easily obtains after simplification

$$
\mathrm{C}_{t^{n}}(g(t))^{k}=k\left(\begin{array}{c}
2 n-k \\
n
\end{array}\right) \sum_{j=1}^{n-k} \frac{(-1)^{j}}{n+j}\left(\begin{array}{c}
n-k \\
j
\end{array}\right) \mathbf{C}_{t^{n-k+j}}(f(t))^{j}
$$

Making use of (2), the relation (16) may be expressed in terms of $A_{1}(\cdot, \cdot)$ and $A_{2}(\cdot, \cdot)$. In fact, simple computations show that (16) is precisely equivalent to (14) with $(i, j)=(2,1)$. Evidently $(14)$ also holds for $(i, j)=(1,2)$ since $g(t)$ and $f(t)$ are interchangeable. This proves that (12) implies (14) via (13).

It remains to show that (14) implies (12). Clearly it suffices to verify that (16) implies (12). Since $\phi(t)=f(t) / t$ is a power series, it is plain that one can compute the right-hand side of (16) by means of (13) as follows

$$
\begin{aligned}
k\left(\begin{array}{c}
2 n-k \\
n
\end{array}\right) \sum_{j=1}^{n-k} \frac{(-1)^{j}}{n+j}\left(\begin{array}{c}
n-k \\
j
\end{array}\right) \mathrm{C}_{t^{n-k}}(\phi(t))^{j} & =\frac{k}{n} \frac{1}{(n-k) !} n\left(\begin{array}{c}
2 n-k \\
n
\end{array}\right)^{n-k} \sum_{j=1}^{n-k} \frac{(-1)^{j}}{n+j}\left(\begin{array}{c}
n-k \\
j
\end{array}\right)\left(\frac{d}{d t}\right)_{0}^{n-k}(\phi(t))^{j} \\
& =\frac{k}{n} \frac{1}{(n-k) !}\left(\frac{d}{d t}\right)_{0}^{n-k}(\phi(t))^{-n} \\
& =\frac{k}{n} \mathbf{C}_{t^{n-k}}\left(\frac{f(t)}{t}\right)^{-n}
\end{aligned}
$$

ithis gives precisely the right-hand side of (12), thus completing the proof of the theorem. 
Certainly the general reciprocal formula (14) of Schlömilch applies to every special Stirling-type pair as displayed in the table of Section 1.

Example. From the table of Section 1 it is seen that the central factorial numbers $t_{1}(n, k)$ of the first kind may be defined by the exponential generating function

$$
\frac{1}{k !}\left[2 \operatorname{arcsinh}\left(\frac{t}{2}\right)\right]^{k}=\sum_{n \geqq 0} t_{1}(n, k) \frac{t^{n}}{n !}
$$

It was mentioned in a recent paper [4] by Butzer, Markett and Schmidt that "one of the most fascinating features of these numbers is the role they play in representing the Riemann zeta function at integer arguments, etc.". For example, they obtained the following interesting formula for $m \geqq 1$ :

$$
\zeta(2 m+1)=2 \sum_{k=1}^{m} \sum_{j=k}^{\infty} \frac{t_{1}(2 j, 2 k)}{j^{2 m-2 k+1}(2 j) !}+\frac{1}{2} \sum_{j=m}^{\infty} \frac{t_{1}(2 j, 2 m)}{j(2 j) !} .
$$

This indicates that a general expression for $t_{1}(n, k)$ should be desired. In fact, it has been found that (cf. [3] also [4])

$$
t_{1}(n, k)=(-1)^{n+k} \sum_{j=k}^{n}\left(\begin{array}{l}
j-1 \\
k-1
\end{array}\right)\left(\frac{n}{2}\right)^{j-k} S_{1}(n, j)
$$

However, this formula as it stands actually consists of a triple summation of elementary terms, inasmuch as the expression for $S_{1}(n, j)$ is of rank two.

Now let us apply the Schlömilch formula (14) to $t_{1}(n, k)$, obtaining

$$
t_{1}(n, k)=\sum_{v=0}^{n-k}(-1)^{v}\left(\begin{array}{c}
2 n-k \\
n-k-v
\end{array}\right)\left(\begin{array}{l}
n-1+v \\
n-k+v
\end{array}\right) t_{2}(n-k+v, v)
$$

Here in accordance with (2) one may compute $t_{2}(n, k)$ via the expression

$$
t_{2}(n, k)=\frac{1}{k !}\left(\frac{d}{d t}\right)_{0}^{n}\left(2 \sinh \frac{t}{2}\right)^{k}
$$

By performing the differentiation one can find a formula of rank one:

$$
t_{2}(n, k)=\frac{1}{k !} \sum_{j=0}^{k}(-1)^{k+j}\left(\begin{array}{l}
k \\
j
\end{array}\right)\left(j-\frac{k}{2}\right)^{n}
$$

Thus, combining (20) with (21), one obtains a formula of rank two as follows 


$$
t_{1}(n, k)=\sum_{v=0}^{n-k} \sum_{j=0}^{v} \frac{(-1)^{j}}{v !}\left(\begin{array}{l}
v \\
j
\end{array}\right)\left(\begin{array}{c}
2 n-k \\
n-k-v
\end{array}\right)\left(\begin{array}{l}
n-1+v \\
n-k+v
\end{array}\right)\left(j-\frac{v}{2}\right)^{n-k+v, v}
$$

This is simpler than (19), and similarly to the case of (11), it may be the "shortest formula" for $t_{1}(n, k)$.

\section{Congruence relations}

The general Schlömilch formula can be used to investigate some divisibility properties of Stirling-type pairs of numbers.

Let $A_{1}(n, k)$ and $A_{2}(n, k)$ be defined by (1). From the Schlömilch formula (14) it is clear that all the numbers $A_{1}(n, k)$ are integers if all $A_{2}(n, k)$ are integers, and vice versa. What we are now going to prove is the following.

Theorem 2. Suppose that all the numbers $A_{1}(n, k)$ as well as $A_{2}(n, k)$ are integers. Let $p$ be an odd prime number and $k$ be any integer such that $1<k<p \leqq 2 k-1$. Then the congruence relations

$$
A_{i}(p+j, k+j) \equiv 0 \quad(\bmod p),(i=1,2)
$$

hold for all $j(0 \leqq j \leqq p-k)$.

Proof. Using the Schlömilch formula we have

$$
A_{1}(p+j, k+j)=\sum_{v=1}^{p-k}(-1)^{v}\left(\begin{array}{c}
2 p+j-k \\
p-k-v
\end{array}\right)\left(\begin{array}{c}
p+j-1+v \\
p-k+v
\end{array}\right) A_{2}(p-k+v, v)
$$

where the first term containing $A_{2}(p-k+0,0)=0$ has been deleted. Note that the condition $1<k<p \leqq 2 k-1$ implies $1 \leqq p-k \leqq k-1$.

From (24) we see that in order to prove (23) with $i=1$, it suffices to show that the congruence relation

$$
\left(\begin{array}{c}
p+j+v-1 \\
p-k+v
\end{array}\right) \equiv 0 \quad(\bmod p)
$$

holds for all $v(1 \leqq v \leqq p-k \leqq k-1)$ and $j(0 \leqq j \leqq p-k)$. In fact, the other binomial coefficient factor appearing in the summation of the right-hand side of (24) will take the value 1 when $v=p-k$, so it cannot be divisible by $p$ for all possible $v$.

$$
\left(\begin{array}{c}
p+j+v-1 \\
k+j-1
\end{array}\right)=\frac{1}{(k+j-1) !} \prod_{s=1}^{k+j-1}(p+v+j-s)
$$


Evidently, for all $j(0 \leqq j \leqq p-k),(k+j-1) \leqq k+(p-k)-1=p-1$, so we have

$$
(k+j-1) ! \neq 0 \quad(\bmod p)
$$

On the other hand, the greatest factor of the product contained in the right-hand side of (26) is seen to be $(p+v+j-1) \geqq p+j \geqq p$ and the least factor is given by $(p+v+j-(k+j-1))=p+v-(k-1) \leqq p$ for all $v(1 \leqq v \leqq k-1)$. Consequently we obtain

$$
\prod_{s=1}^{k+j-1}(p+v+j-s) \equiv 0 \quad(\bmod p)
$$

Hence (25) is true for all $v$ and $j$, and this completes the proof of the theorem since $A_{1}$ and $A_{2}$ of (24) are interchangeable.

Examples. It is known that the tangent and arctangent numbers $T_{1}(n, k)$ and $T_{2}(n, k)$ as well as the classical Stirling numbers are all integers. Thus as an immediate consequence of Theorem 2 we may have the congruence relations

$$
\begin{array}{ccc}
T_{i}(p+j, k+j) \equiv 0 & (\bmod p), & (i=1,2) \\
S_{i}(p+j, k+j) \equiv 0 & (\bmod p), & (i=1,2)
\end{array}
$$

where $p$ is any prime number such that $1<k<p \leqq 2 k-1$, and $j$ ranges over the interval $[0, p-k]$.

Remark. For the kinds of Stirling numbers it is well-known that there hold the congruence relations $S_{i}(p, k) \equiv 0(\bmod p),(1=1,2)$, for all $k(1<k<p)$, (cf. [5, §5.8]). However, we are inclined to think that the general congruence relations $A_{i}(p, k) \equiv 0$ $(\bmod p)(i=1,2)$ may not hold without the restriction $p \leqq 2 k-1$, if all $A_{i}(p, k)$ are integers.

\section{Asymptotic expansions}

Here we wish to obtain a kind of asymptotic expansion for the general Stirling-type pair $A_{i}(n, k)(i=1,2)$ involving large parameters $n$ and $k$ such that $r=n-k$ is fixed or $r=o(\sqrt{n})(n \rightarrow \infty)$. This will be achieved by the aid of a general asymptotic formula which was established in our recent paper [8].

Let $\sigma(n)$ denote the set of partitions of $n \in Z_{+}$, usually represented by $1^{k_{1}} 2^{k_{2}} \ldots n^{k_{n}}$ with $k_{1}+2 k_{2}+\cdots n k_{n}=n, k_{i}$ being the number of parts of size $i$. Moreover, denote by $\sigma(n, k)$ the subset of $\sigma(n)$ consisting of the partitions of $n$ with $k$ parts, namely

$$
\sigma(n, k):=\left\{1^{k_{1}} 2^{k_{2}} \ldots n^{k_{n}}: \sum_{i=1}^{n} i k_{i}=n, \sum_{i=1}^{n} k_{i}=k\right\},
$$


Let $\phi(t)$ be a formal power series over the complex field $C$ with $\phi(0)=1$. Then for every number $w \in C$ one may write the formal series expansion of $(\phi(t))^{w}$ in the most convenient form

$$
(\phi(t))^{w}=\sum_{n \geqq 0}\left\{\begin{array}{l}
w \\
n
\end{array}\right\} t^{n}
$$

Certainly, $\left\{\begin{array}{ll}w & n\end{array}\right\}$ may also be expressed using the notation of Bell polynomials or potential polynomials (cf. [5, chap, 3]). In particular, $\left\{\begin{array}{ll}w & 0\end{array}\right\}=(\phi(0))^{\omega}=1$. What we will need is the following result (cf. [8]).

For any $\alpha \in \mathbf{C}$ and every integer $j(0 \leqq j<n)$ denote

$$
W(\alpha, n j)=\sum_{\sigma(n, n-j)} \prod_{i=1}^{n} \frac{1}{k_{i} !}\left\{\begin{array}{l}
\alpha \\
i
\end{array}\right\}^{k_{i}}
$$

where the summation is taken over all the partitions $1^{k_{1}} 2^{k_{2}} \ldots n^{k_{n}}$ belonging to the set $\sigma(n, n-j)$. Then for every given $m<n$ and for $|\lambda| \rightarrow \infty$ with $\lambda \in \mathbf{C}$ we have an asymptotic expansion of the form

$$
\frac{1}{(\lambda)_{n}}\left\{\begin{array}{c}
\lambda \alpha \\
n
\end{array}\right\}=\sum_{j=0}^{m} \frac{W(\alpha, n, j)}{(\lambda-n+j)_{j}}+O\left(\left(\frac{1}{\lambda}\right)^{m+1}\right)
$$

where $(\lambda)_{n}=\lambda(\lambda-1) \ldots(\lambda-n+1)$ is the falling factorial.

In view of the definition for the set $\sigma(n, k)$, it is easily found that $W(\alpha, n, j)$ consists of only a few terms when $j=0,1,2,3$. For instance, $W(\alpha, n, 3)$ is a sum of 3 terms

$$
W(\alpha, n, 3)=\frac{1}{(n-4) !}\left\{\begin{array}{l}
\alpha \\
1
\end{array}\right\}^{n-4}\left\{\begin{array}{l}
\alpha \\
4
\end{array}\right\}+\frac{1}{(n-5) !}\left\{\begin{array}{l}
\alpha \\
1
\end{array}\right\}^{n-5}\left\{\begin{array}{l}
\alpha \\
2
\end{array}\right\}\left\{\begin{array}{l}
\alpha \\
3
\end{array}\right\}+\frac{1}{3 !(n-6) !}\left\{\begin{array}{l}
\alpha \\
1
\end{array}\right\}^{n-6}\left\{\begin{array}{l}
\alpha \\
2
\end{array}\right\}^{3}
$$

and consequently for fixed $\alpha$

$$
W(\alpha, n, 3)=\frac{1}{3 !(n-6) !}\left\{\begin{array}{l}
\alpha \\
1
\end{array}\right\}^{n-6}\left\{\begin{array}{l}
\alpha \\
2
\end{array}\right\}^{3}\left[1+O\left(\frac{1}{n}\right)\right], \quad(n \rightarrow \infty)
$$

Evidently, $W(\alpha, n, j)$ will become more and more complicated as $j$ increases. However we have

$$
W(\alpha, n, j)=\frac{1}{j !(n-2 j) !}\left\{\begin{array}{l}
\alpha \\
1
\end{array}\right\}^{n-2 j}\left\{\begin{array}{l}
\alpha \\
2
\end{array}\right\}^{j}\left[1+O\left(\frac{1}{n}\right)\right]
$$


This may be useful for estimating $W(\alpha, n, j)$ when $n$ is large.

Let $A_{r}(n, k)(r=1,2)$ be defined by (1). In order to make (30) applicable to $A_{r}(n, k)$ we have to consider expansions

$$
\left(\phi_{1}(t)\right)^{k}=\sum_{n \geqq 0} \frac{A_{1}(n+k, k)}{(n+k)_{n}} t^{n}, \quad\left(\phi_{2}(t)\right)^{k}=\sum_{n \geqq 0} \frac{A_{2}(n+k, k)}{(n+k)_{n}} t^{n},
$$

where $\phi_{1}(t)=f(t) / t$ and $\phi_{2}(t)=g(t) / t$ satisfy the required condition $\phi_{1}(0)=\phi_{2}(0)=1$.

In what follows we assume $\lambda \in \mathbf{Z}_{+}$. Comparing (32) with (28) we find

$$
\left\{\begin{array}{l}
\lambda \\
n
\end{array}\right\}=\frac{A_{r}(\lambda+n, \lambda)}{(\lambda+n)_{n}} . \quad(\mathrm{r}=1,2)
$$

Hence, making use of (30) with $\alpha=1$ we are led to the following theorem.

Theorem 3. Given any $n \in \mathbf{Z}_{+}$, then for $\lambda$ large we have

$$
\frac{A_{r}(\lambda+n, \lambda)}{(\lambda)_{n}(\lambda+n)_{n}}=\sum_{j=0}^{s} \frac{W_{r}(n, j)}{(\lambda-n+j)_{j}}+O\left(\left(\frac{1}{\lambda}\right)^{s+1}\right)
$$

where $s<n$ and $W_{r}(n, j)(r=1,2)$ are defined by

$$
W_{r}(n, j)=\sum_{\sigma(n, n-j)} \prod_{i=1}^{n} \frac{1}{k_{i} !}\left(\frac{A_{r}(i+1,1)}{(i+1) !}\right)^{k_{i}}
$$

Remark. Worth noticing is that (31) implies the following

$$
\frac{W(\alpha, n, j+1)}{(\lambda-n+j+1)_{n+1}}=O\left(\frac{n^{2} W(\alpha, n, j)}{\lambda(\lambda-n+j)_{j}}\right)
$$

Thus one can find that the asymptotic formula (30) can be extended to the case when $|\lambda| \rightarrow \infty$ and $n \rightarrow \infty$ with $n=o\left(|\lambda|^{1 / 2}\right)$, but the error term $O\left((1 / \lambda)^{m+1}\right)$ should be replaced by $o\left(W(\alpha, n, m) /(\lambda-n+m)_{m}\right)$. Consequently, (34) has an extended form of the following

$$
\frac{A_{r}(\lambda+n, \lambda)}{(\lambda)_{n}(\lambda+n)_{n}}=\sum_{j=0}^{s} \frac{W_{r}(n, j)}{(\lambda-n+j)_{j}}+o\left(\frac{W_{r}(n, s)}{(\lambda-n+s)_{s}}\right)
$$

where $s<n$ and $n=o(\sqrt{\lambda})(\lambda \rightarrow \infty)$.

Examples. For $A_{r}(\lambda+n, \lambda):=S_{r}(\lambda+n, \lambda), T_{r}(\lambda+n, \lambda), t_{r}(\lambda+n, \lambda)(r=1,2)$ one can make 
use of either (34) or (36) to get six special asymptotic expansions for $S_{r}, T_{r}$, and $t_{r}$ respectively, where the error terms to be estimated may be $O\left((1 / \lambda)^{\text {st }}\right)$ or $o\left(W_{r}\left((n, s) /(\lambda-n+s)_{s}\right)\right.$ according as $n$ is fixed or $n=o(\sqrt{\lambda}), \lambda$ being large. Note that the computation of (35) for Stirling numbers may become more simplified since $S_{1}(i+1,1)=$ $(-1)^{i} i$ ! and $S_{2}(i+1,1)=1$. In fact, the asymptotic expansion of $S_{2}(\lambda+n, \lambda)$ implied by (34) (or 36)) is substantially equivalent to the old one found by the author [9] in 1948 (cf. also [11]). It is known that a useful extension of the asymptotic formula for Stirling functions of the second kind was obtained by Butzer and Hauss [2].

\section{REFERENCES}

1. P. L. Butzer and M. Hauss, Stirling functions of the first and second kind; some new applications, Approximation, Interpolation and Summability (Proc. Conf. in honour of Prof. Jakimovski, Tel Aviv, June 4-8, 1990, Research Institute of Math. Sciences, Bar Ilan Univ., Weizmann Press, Israel).

2. P. L. Butzer and M. Hauss, On Stirling functions of the second kind, preprint from Lehrstuhl A für Mathematik, Aachen Univ. of Technology, 1989.

3. P. L. Butzer, M. Schmidt, E. L. Stark and L. Vogt, Central factorial numbers; their main properties and some applications, Numer. Funct. Anal. Optim. 10 (1989), 419-488.

4. P. L. Butzer, C. Markett and M. Schmidt, Stirling numbers, central factorial numbers, and representations of the Riemann zeta function, preprint from Lehrstuhl A für Mathematik, Aachen Univ. of Technology, 1991.

5. L. Сомтет, Advanced Combinatorics (D. Reidel Publ. Company, Dordrecht, Holland, 1974).

6. L. C. Hsu, Generalized Stirling number pairs associated with inverse relations, Fibonacci Quart. 25 (1987), 346-351.

7. L. C. Hsu, Theory and application of generalized Stirling number pairs, J. Math. Res. Exposition Quart. 9 (1989), 211-220.

8. L. C. Hsu and W. CHu, A kind of asymptotic expansion using partitions, Tôhoku Math. J. 43 (1991), 235-242.

9. L. C. Hsu, Note on an asymptotic expansion of the nth difference of zero, Ann Math. Stat. 19 (1948), 273-277.

10. C. Jordan, Calculus of Finite Differences (Chelsea Publ. Company, New York, 1950).

11. L. Moser and M. Wyman, Stirling numbers of the second kind, Duke Math. J. 25 (1958), $29-43$.

Institute of Mathematical Sciences

Dalian University of Technology

DaLIAN 116024

China 